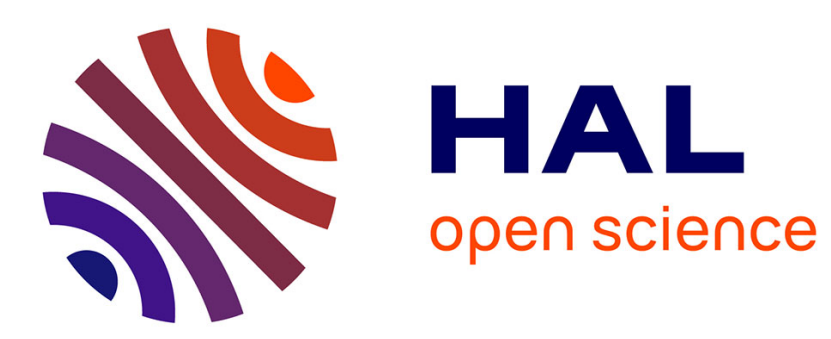

\title{
Global sensitivity analysis for modeling the free-flight behavior of an artillery projectile
}

Dawid Machala, Floriane Collin, Marion Gilson, Marie Albisser, Simona Dobre

\section{To cite this version:}

Dawid Machala, Floriane Collin, Marion Gilson, Marie Albisser, Simona Dobre. Global sensitivity analysis for modeling the free-flight behavior of an artillery projectile. AIAA Journal, 2020, 58 (7), pp.3139-3148. 10.2514/1.J059065 . hal-02892171

\section{HAL Id: hal-02892171 \\ https://hal.science/hal-02892171}

Submitted on 7 Jul 2020

HAL is a multi-disciplinary open access archive for the deposit and dissemination of scientific research documents, whether they are published or not. The documents may come from teaching and research institutions in France or abroad, or from public or private research centers.
L'archive ouverte pluridisciplinaire HAL, est destinée au dépôt et à la diffusion de documents scientifiques de niveau recherche, publiés ou non, émanant des établissements d'enseignement et de recherche français ou étrangers, des laboratoires publics ou privés. 


\title{
Global Sensitivity Analysis for Modeling the Free-Flight Behavior of an Artillery Projectile
}

\author{
Dawid Machala* \\ French-German Research Institute of Saint Louis (ISL), 5 Rue du Général Cassagnou, 68301, France \\ Université de Lorraine, CNRS, CRAN, F-54000 Nancy, France \\ Floriane Collin ${ }^{\dagger}$ and Marion Gilson ${ }^{\dagger}$ \\ Université de Lorraine, CNRS, CRAN, F-54000 Nancy, France \\ Marie Albisser ${ }^{\S}$ and Simona Dobre ${ }^{\mathbb{I I}}$ \\ French-German Research Institute of Saint Louis (ISL), 5 Rue du Général Cassagnou, 68301, France
}

A vehicle's in-flight behavior can be represented by the Newton-Euler equations of motion: usually, such a model has a nonlinear and continuous description based on ordinary differential equations. The model structure can be altered using analytic transformations-such as transformation into a quasi-LPV structure-and, when the model is supposed to reflect an existing physical system, some of its parameters might be uncertain. This paper's objective is twofold: firstly, to investigate if a quasi-LPV model does accurately replicate the physical behavior of a large-caliber spin-stabilized projectile in free flight; and secondly, to verify if the available embedded sensors data could be used to determine non-identifiability of uncertain model parameters. In order to investigate these prospects, a permutation-based global sensitivity analysis is employed. The proposed study has highlighted the non-identifiable parameters of the model, either during the whole flight or some periods of it. These results can help to enhance the preparation of free flight experiments at the open-range test site of the French-German Research Institute of Saint Louis.

\section{Nomenclature}




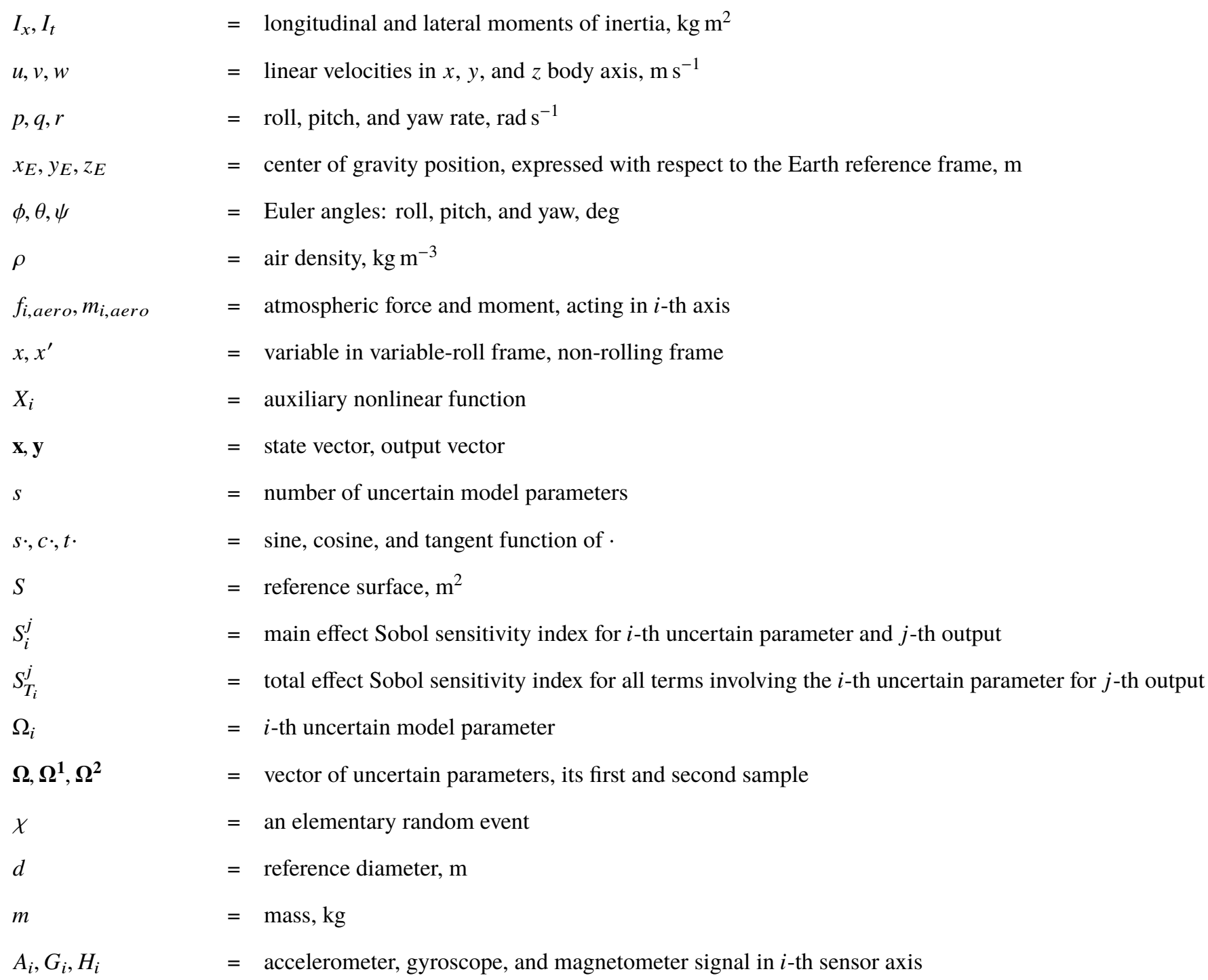

\section{Introduction}

N important trend in the development of $155 \mathrm{~mm}$ artillery ammunition aims towards higher precision and longer
range, especially through the use of various guidance and navigation techniques to assure their capability of hitting even a small or moving target. Such a development relies on a well-investigated and -understood mathematical description of a projectile's behavior. A first-principles' model of a projectile is described by nonlinear Newton-Euler laws of motion, and depends on a set of aerodynamic coefficients that need to be quantified for each flying vehicle [1]. In other words, the knowledge of the aerodynamic characterisation allows to predict the trajectory and assess the dynamical behavior of a projectile, hence underlying the importance of their quantification.

The aerodynamic coefficients can be quantified using several complementary methods, such as Computational Fluid Dynamics simulations (e.g. in [2]), semi-empirical codes (e.g. PRODAS [3] and Missile DATCOM [2]), or wind-tunnel 
experiments (e.g. in [4]). Their results then serve as a first guess of the aerodynamic coefficients, later to be confirmed under a real-world experimentation—ballistic free-flight tests [5].

Free-flight tests pose several technical and scientific difficulties. It must be assured that the projectile will land in a dedicated safe zone, even though some of the initial state variables are unknown; or that the identified model structure will be useful for the guidance and navigation tasks, even though the physical model is nonlinear. Moreover, due to the fact that the experiments are performed in real-flight conditions, several sources of uncertainty are introduced to model parameters. For instance, initial state vector will differ from its nominal value due to stochastic effects induced by interior ballistics [6]. Similarly, an initial guess of the aerodynamic coefficients, based on similar architectures in the literature, will likely be imprecise. These uncertainties influence the model dynamics, and thus propagate through the model into its output signals.

The influence that the uncertain model parameters have on the model output can be assessed by the means of sensitivity analysis that apportions the output uncertainty to its different sources [7]. Such a knowledge offers the possibility of evaluating the influence of parameter uncertainties on the model behavior [8-10]. When assessed, the 'least' influential parameters can be set to a nominal value, since they do not significantly affect the model dynamics, whereas the identification and modelling efforts can be concentrated on the most influential parameters. The methods of sensitivity analysis can be divided into local and global approaches. The local technique assess the uncertain parameters' influence around nominal operating points [8], primarily using one-at-a-time sampling and differential analysis [11]. However, they fail to characterize the behavior of the model far from the selected nominal condition, and—in the case of one-at-the-time sampling — explore only a limited area of the state-space [7]. The global methods aim to explore the full space of uncertain model parameters. There are several strategies to assess the global sensitivity of the model. Principally the variance-based analysis using so-called Sobol' indices is employed (also called Analysis of Variance, ANOVA) [7, 12], which allows to determine both the individual influence of each parameter and their interactions [7, 13].

In aerospace applications, sensitivity analysis is usually conducted using local sensitivity analysis, as in [14-19]. Only a few works deal with global sensitivity analysis (GSA). Among them, the prominent applications include the works of Huan et al. [20] who used a surrogate modeling approach to identify the most influential parameters in scramjet combustor simulations. Wei et al. [21] employed a modified Monte-Carlo approach to calculate sensitivity indices in order to minimize the variance of model output for a wing-structure model. Jiang et al. [22] have used GSA in order to guide a design process of an aircraft by assessing which model parameters influence the most its final acquisition cost or maximum flight time. It is worth noting that the results of a local sensitivity analysis can be misleading or even significantly different than of the global study. For example, Hale et al. [23] have shown that local sensitivity analysis might wrongly indicate some of the model parameters as influential; as well as it significantly underestimates the influence of aerodynamic coefficients due to their nonlinearity. However, to authors' knowledge, only local sensitivity 
analysis has been applied in the existing studies of free-flight experiments in the domain of exterior ballistics.

In the context of the aforementioned experimental and practical difficulties associated with free-flight experiments, the authors investigate the possibilities of enhancing the traditional procedure for conducting the tests by the use of GSA. Potentially, this technique could be used as an effective simulation-based tool of visualizing physical knowledge in ballistic systems and detecting non-identifiable model parameters. Moreover, this technique could be seen as an simulation-based alternative to observability analysis and information theory, that are sometimes used in exterior ballistics to detect which model parameters should be identified, as shown, e.g. in [24-26].

The following provides the problem statement: to attribute the sources of uncertainties on the simulated embedded sensor signals to the uncertain model parameters using GSA. For this purpose, a first-principles-based model has been transformed into a quasi-linear parameter varying (LPV) model of lower computational complexity. Such a model structure is selected due to its prevalence in the domain of control and navigation. GSA is then performed on the resulting quasi-LPV model. It is then checked if the implications of the sensitivity indices on the identifiability of uncertain parameters are coherent with the domain knowledge of exterior ballistics. The quasi-LPV model structure has been recently presented [27] and its accuracy has been numerically confirmed [28].

The structure of the paper is as follows. In section II, the nonlinear and quasi-LPV model structures are introduced, and the sources of their uncertainty are explained. Section III provides an overview of the theory behind the GSA whereas its results when applied to the quasi-LPV model are summarized in section IV. Section V concludes the article by summarizing the main contributions and future prospects of this work.

\section{Modeling}

The nonlinear model, based on the 6-degrees-of-freedom Newton-Euler laws of motion [29], and its representation in a quasi-LPV form [27], are presented hereafter. Both models assume that the mass distribution is uniform and that the shape of projectile is axis-symmetrical.

\section{A. Nonlinear model}

The model of the in-flight dynamics is a nonlinear state-space continuous-time structure $\mathcal{M}$ :

$$
\mathcal{M}:\left\{\begin{array}{l}
\dot{\mathbf{x}}(t)=f\left(\mathbf{x}(t), \mathbf{C}\left(\mathbf{x}(t), \mathbf{p}_{a}\right)\right), \quad \mathbf{x}(0)=\mathbf{x}_{0} \\
\mathbf{y}(t)=g(\mathbf{x}(t)),
\end{array}\right.
$$

where the state vector $\mathbf{x} \in \mathbb{R}^{12}$ has 12 components corresponding to the translational velocities $(u, v, w)$, rotational velocities $(p, q, r)$, position of the center of gravity of the vehicle with respect to Earth $\left(x_{E}, y_{E}, z_{E}\right)$, and the roll, pitch and yaw angles ( $\phi, \theta$ and $\psi$, respectively) [1]. The aerodynamic coefficients $\mathbf{C}$ are described by polynomial functions 
whose time invariant parameters are $\mathbf{p}_{a}$. These descriptions depend on the linear and angular velocities, as well as on the Mach number $M$ and the total angle of attack $\alpha_{t}$, which are defined by:

$$
M=\frac{V}{a}, \quad \alpha_{t}=\arccos \left(\frac{u}{V}\right),
$$

where $a$ is the speed of sound, and $V$ is the total velocity, i.e. $V=\sqrt{u^{2}+v^{2}+w^{2}}$ [29]. The output vector $\mathbf{y}$ represents the data obtained by on-board and on-ground measurement techniques available during free flight tests. Herein, embedded magnetometers, accelerometers and gyroscopes are considered.

The full form of the nonlinear model equations in the variable-roll reference frame is described by the following set of equations [29]:

\section{Translational dynamics}

$$
\left[\begin{array}{c}
\dot{u} \\
\dot{v} \\
\dot{w}
\end{array}\right]=\left[\begin{array}{ccc}
0 & -r & q \\
r & 0 & -p \\
-q & p & 0
\end{array}\right]\left[\begin{array}{l}
u \\
w
\end{array}\right]+g\left[\begin{array}{l}
-s \theta \\
s \phi c \theta \\
c \phi c \theta
\end{array}\right]+1 / m\left[\begin{array}{l}
f_{x, \text { aero }} \\
f_{y, \text { aero }} \\
f_{z, \text { aero }}
\end{array}\right],
$$

Attitude dynamics

$$
\left[\begin{array}{c}
\dot{p} \\
\dot{q} \\
\dot{r}
\end{array}\right]=\left[\begin{array}{c}
0 \\
p \cdot r \frac{I_{t}-I_{x}}{I_{t}} \\
p \cdot q \frac{I_{x}-I_{z}}{I_{t}}
\end{array}\right]+\left[\begin{array}{c}
\frac{1}{I_{x}} m_{x, \text { aero }} \\
\frac{1}{I_{y}} m_{y, \text { aero }} \\
\frac{1}{I_{z}} m_{z, \text { aero }}
\end{array}\right],
$$

Translational kinematics

$$
\left[\begin{array}{c}
\dot{x}_{E} \\
\dot{y}_{E} \\
\dot{z}_{E}
\end{array}\right]\left[\begin{array}{ccc}
c \theta c \psi & s \phi s \theta c \psi-s \psi c \phi & c \phi s \theta c \psi+s \phi s \psi \\
c \theta s \psi & s \phi s \theta s \psi+c \psi c \phi & c \phi s \theta s \psi-s \phi c \psi \\
-s \theta & s \phi c \theta & c \phi c \theta
\end{array}\right]\left[\begin{array}{c}
u \\
v \\
w
\end{array}\right],
$$

Attitude kinematics

$$
\left[\begin{array}{l}
\dot{\phi} \\
\dot{\theta} \\
\dot{\psi}
\end{array}\right]=\left[\begin{array}{ccc}
1 & s \phi t \theta & c \phi t \theta \\
0 & c \phi & -s \phi \\
0 & s \phi \frac{1}{c \theta} & c \phi \frac{1}{c \theta}
\end{array}\right]\left[\begin{array}{l}
p \\
q \\
r
\end{array}\right]
$$


where the forces and moments are given by:

$$
\begin{gathered}
{\left[\begin{array}{l}
f_{x, \text { aero }} \\
f_{y, \text { aero }} \\
f_{z, \text { aero }}
\end{array}\right]=\bar{q} S\left[\begin{array}{c}
C_{X_{0}}+C_{X_{a}} s^{2} \alpha_{t} \\
-C_{y p \alpha} \frac{d}{2 V} \frac{w}{V} p-C_{N \alpha} \frac{v}{V} \\
-C_{y p \alpha} \frac{d}{2 V} \frac{v}{V} p-C_{N \alpha} \frac{w}{V}
\end{array}\right],} \\
{\left[\begin{array}{c}
m_{x, \text { aero }} \\
m_{y, \text { aero }} \\
m_{z, \text { aero }}
\end{array}\right]=\bar{q} S d\left[\begin{array}{c}
C_{l p} p \\
C_{m \alpha} \frac{w}{V}-C_{n p \alpha} \frac{d}{2 V} \frac{v}{V} p+C_{m q} \frac{d}{2 V} q \\
-C_{m \alpha} \frac{v}{V}-C_{n p \alpha} \frac{d}{2 V} \frac{w}{V} p+C_{m q} \frac{d}{2 V} r
\end{array}\right] .}
\end{gathered}
$$

The state equations depend on various physical properties, measured or determined for each projectile before the flight tests, such as the mass $m$, longitudinal and lateral moments of inertia $I_{x}, I_{t}$, reference surface $S$, and diameter $d$. The dynamic pressure is denoted by $\bar{q}=\frac{1}{2} \rho V^{2}$, where $\rho$ is the air density, also assumed to be known. Symbols $s, c, t$ correspond to trigonometric sine, cosine, and tangent, respectively.

The aerodynamic coefficients can be divided into the force and moment coefficients. The forces are described via the axial force $C_{X}$ (decomposed as $C_{X}=C_{X_{0}}+C_{X_{a}} s^{2} \alpha_{t}$ ), the normal force slope $C_{N \alpha}$ and Magnus force slope $C_{y p \alpha}$ coefficients. Similarly, the moments are expressed through the roll damping $C_{l p}$, pitch moment coefficient slope $C_{m \alpha}$, pitch damping $C_{m q}$, and Magnus moment slope $C_{n p \alpha}$ coefficients [29].

\section{B. Quasi-LPV model}

The proposed quasi-LPV model is based on analytic function substitution applied to the nonlinear model equations. In order to simplify the computational complexity of the nonlinear model, and to aid the analytic substitution process, a coordinate-system transformation has been applied to the nonlinear model equations.

In aerospace modeling, the usual choice for a coordinate system location is to rigidly fix it on the vehicle's center of mass and to subject it to all the projectile's motions [1,30]. Alternatively, the model can be developed with respect to a frame that is fixed to the projectile's center of mass, but does not experience the rolling motion [30]. Such descriptions are called "variable-roll" and "non-rolling" reference frames, respectively. The latter formulation has an interesting property if the projectile is axis-symmetric (in $y$ and $z$ axes) and spin-stabilized: the nonlinear model equations become decoupled from its fastest changing variable, the roll angle $\phi[30]$. As a result, the computational complexity diminishes. The non-rolling frame has been applied for this purpose as early as in 1960s (e.g. in [31]).

Transformation of dynamics state variables in variable-roll reference frame (3)-(4) into their non-rolling equivalents 
can be described using the following transformation equations as:

$$
\left[\begin{array}{c}
u^{\prime} \\
v^{\prime} \\
w^{\prime}
\end{array}\right]=\left[\begin{array}{ccc}
1 & 0 & 0 \\
0 & c \phi & -s \phi \\
0 & s \phi & c \phi
\end{array}\right]\left[\begin{array}{l}
u \\
v \\
w
\end{array}\right], \quad\left[\begin{array}{l}
p^{\prime} \\
q^{\prime} \\
r^{\prime}
\end{array}\right]=\left[\begin{array}{ccc}
1 & 0 & 0 \\
0 & c \phi & -s \phi \\
0 & s \phi & c \phi
\end{array}\right]\left[\begin{array}{l}
p \\
r \\
r
\end{array}\right],
$$

where the superscript $\cdot{ }^{\prime}$ indicates a variable expressed with respect to the non-rolling frame. The kinematic state variables, i.e. Euler angles and positions, are equivalent in both frames.

A quasi-LPV model can be obtained in a non-rolling frame through a set of algebraic transformations, in accordance with the function substitution approach [32]. The full derivation of the model has been presented in [27], and results in the following set of equations:

$$
\dot{\mathbf{x}}^{\prime}=A\left(\mathbf{x}^{\prime}\right) \cdot \mathbf{x}^{\prime},
$$

where the non-rolling state vector $\mathbf{x}^{\prime}=\left[\begin{array}{lllllllllll}u^{\prime} & v^{\prime} & w^{\prime} & p^{\prime} & q^{\prime} & r^{\prime} & \theta^{\prime} & \psi^{\prime} & x_{E}^{\prime} & y_{E}^{\prime} & z_{E}^{\prime}\end{array}\right]^{T} \in \mathbb{R}^{11}$, and the parameter-

\begin{tabular}{|c|c|c|c|c|c|c|c|c|c|}
\hline & 0 & $-r^{\prime}$ & $q^{\prime}$ & $\frac{X_{1}\left(u^{\prime}, V, z_{E}^{\prime}\right)-g\left(z_{E}^{\prime}\right) s \theta^{\prime}}{p^{\prime}}$ & 0 & 0 & 0 & 0 & 0 \\
\hline & $r^{\prime}$ & $X_{3}\left(V, z_{E}^{\prime}\right)$ & $r^{\prime} t \theta^{\prime}+X_{2}\left(V, z_{E}^{\prime}\right) p^{\prime}$ & 0 & 0 & 0 & 0 & 0 & 0 \\
\hline & $-q^{\prime}$ & $-r^{\prime} t \theta^{\prime}-X_{2}\left(V, z_{E}^{\prime}\right) p^{\prime}$ & $X_{3}\left(V, z_{E}^{\prime}\right)$ & $\frac{g\left(z_{E}^{\prime}\right) c \theta}{p^{\prime}}$ & 0 & 0 & 0 & 0 & 0 \\
\hline & 0 & 0 & 0 & $X_{4}\left(V, z_{E}^{\prime}\right)$ & 0 & 0 & 0 & 0 & 0 \\
\hline & 0 & $X_{7}\left(V, z_{E}^{\prime}\right) p^{\prime}$ & $X_{5}\left(V, z_{E}^{\prime}\right)$ & $-\frac{I_{x}}{I_{s}} r^{\prime}-\frac{r^{\prime} r^{\prime} t \theta}{p}$ & $X_{6}\left(V, z_{E}^{\prime}\right)$ & 0 & 0 & 0 & 0 \\
\hline$A(x)=$ & 0 & $-X_{5}\left(V, z_{E}^{\prime}\right)$ & $X_{7}\left(V, z_{E}^{\prime}\right) p^{\prime}$ & $\frac{I_{x}}{I_{s}} q^{\prime}+\frac{q^{\prime} r^{\prime} t \theta}{p}$ & 0 & $X_{6}\left(V, z_{E}^{\prime}\right)$ & 0 & 0 & 0 \\
\hline & 0 & 0 & 0 & 0 & 1 & 0 & 0 & 0 & 0 \\
\hline & 0 & 0 & 0 & 0 & 0 & $c^{-1} \theta$ & 0 & 0 & 0 \\
\hline & $c \theta c \psi$ & $-s \psi$ & $s \theta c \psi$ & 0 & 0 & 0 & 0 & 0 & 0 \\
\hline & $c \theta s \psi$ & $c \psi$ & $s \theta s \psi$ & 0 & 0 & 0 & 0 & 0 & 0 \\
\hline & $-s \theta$ & 0 & $c \theta$ & 0 & 0 & 0 & 0 & 0 & 0 \\
\hline
\end{tabular}
dependent state matrix can be defined as follows: 
where:

$$
\begin{aligned}
X_{1}\left(u, V, z_{E}\right) & =-0.5 m^{-1} \rho\left(z_{E}\right) V^{2} S C_{X}(u, V) \\
X_{2}\left(V, z_{E}\right) & =0.25 m^{-1} \rho\left(z_{E}\right) S d C_{y p \alpha}(V) \\
X_{3}\left(V, z_{E}\right) & =-0.5 m^{-1} \rho\left(z_{E}\right) V S C_{N \alpha}(V) \\
X_{4}\left(V, z_{E}\right) & =0.25 \rho\left(z_{E}\right) V S d^{2} C_{l p}(V) \\
X_{5}\left(V, z_{E}\right) & =0.5 \rho\left(z_{E}\right) V S d C_{m \alpha}(V) \\
X_{6}\left(V, z_{E}\right) & =0.25 \rho\left(z_{E}\right) V S d^{2} C_{m q}(V) \\
X_{7}\left(V, z_{E}\right) & =0.25 \rho\left(z_{E}\right) S d^{2} C_{n p a}(V) .
\end{aligned}
$$

The scheduling signals (i.e. the time-varying elements of the state matrix) of the quasi-LPV model consist of eight variables, which means that the model could be considered as a rather complex in control application-especially that the complexity of an LPV controller increases exponentially with the number of variables [33]. However, such a structure can be deemed to be a good compromise between control and identification requirements. Firstly, should the complexity be reduced, fixing some of the scheduling signals will result in a control-oriented structure presented in [34]. Secondly, should the model behavior be accurate for a wide variation of flight conditions, which is necessary for the identification step [29], the model can be left 'as-is'. Out of the eight scheduling signals, five of them can be measured by available measurement techniques: the angular rates by gyroscopes, the altitude by a trajectory tracker, and the total velocity by a Doppler radar. The remaining scheduling signals, i.e. $u^{\prime}, \theta, \psi$, can be simulated or estimated, as in [35].

For the purpose of conducting GSA, the case of a projectile equipped with a set of embedded sensors will be considered in flight simulations. The analysis will be, for the sake of simplicity, shown only for the embedded sensors. Therefore, the following data are analyzed: the signals of axial $A_{x}$ and radial $A_{y}, A_{z}$ accelerometer; radial $G_{y}, G_{z}$ gyroscopes; and axial $H_{x}$ and radial $H_{y}, H_{z}$ magnetometers. These sensors have been previously used and calibrated in ISL's works, and their equations with further details are presented in [29, 36-38]. All of their signals are time-varying.

\section{Sources of model uncertainty}

When a given model structure is simulated, its trajectory depends on the initial state vector $\mathbf{x}_{0}$ and on the set of model parameters, such as its aerodynamic coefficient parameters $\mathbf{p}_{a}$, mass, and inertia. In the following subsections, the set of nominal values of $\mathbf{x}_{0}$ and $\mathbf{p}_{a}$ will be discussed, together with the possible sources of uncertainty influencing them.

\section{Uncertainties of initial state variables}

State variables in the model are divided into these describing translational dynamics, attitude dynamics, translational kinematics, and attitude kinematics. Specifics of each influence the model uncertainty in a different way: 
- Translational dynamics. In the case of free-flight experiments, as performed at the ISL's open-range test site (see [29]), the flights are usually performed over a range of flight conditions (e.g. sub-, trans-, and super-sonic regimes) in order to sufficiently explore the projectile flight dynamics. Herein, a selection of four different initial velocities $u_{0}$, ranging from 300 to $600 \mathrm{~m} \mathrm{~s}^{-1}$ has been chosen as the set of nominal values. Since the projectiles are launched from a rifled-bore powder gun, and each firing varies slightly depending on inaccuracies in powder amount as well as due to internal ballistic effects (such as differences in firing temperature, pressure distribution in-bore, etc [6]), an uncertainty range of $\pm 1 \%$ is assumed. The stochastic effects of internal ballistics on the remaining linear velocities are negligible, therefore $v_{0}, w_{0}$ can be assumed to be zero, without any interval of uncertainty imposed upon them.

- Attitude dynamics. In the case of the initial angular rates, the effects of internal ballistics are non-negligible. The initial roll rate $p_{0}$ is a function of initial axial velocity $u_{0}$ and the twist rate of the launcher's bore [6]. Herein, $p_{0}=2.0283 \cdot u_{0}$ has been assumed, which renders $p_{0}$ a linear transformation of $u_{0}$. Since $u_{0}$ is already considered as an uncertain parameter, no uncertainty is assumed on $p_{0}$. For the initial yaw and pitch rates, the nominal value is $q_{0}=r_{0}=0 \mathrm{rad} \mathrm{s}^{-1}$. However, their nominal values can be influenced by the stochastic uncertainties stemming from the internal ballistics [6]. Due to a lack of more precise estimate, a significant interval of uncertainty of \pm 3 $\operatorname{rad~s}^{-1}$ has been assumed on $q_{0}$ and $r_{0}$.

- Attitude kinematics. The initial position of a projectile is equivalent to the position of the launcher. In this case study, it corresponds to $x_{E_{0}}=y_{E_{0}}=0 \mathrm{~m}$ with the initial altitude of $z_{E_{0}}=-1.5 \mathrm{~m}$, corresponding to the launcher height. Finally, concerning the initial attitude of the projectile, it is dependent on the target location, as the launcher aims to deliver the projectile to its terminal destination, which in the case of ISL's test site corresponds to the centre of a sandbay, located at $[1000,0,-6] \mathrm{m}$. The negative value of the altitude stems from the definition of the Earth reference frame: positive altitude axis aims towards the center of Earth.

- Translational kinematics. The initial attitude of the projectile has to be determined, as it has a direct influence of the trajectory and point of impact. In order to find the initial pitch $\theta_{0}$ and yaw $\psi_{0}$ angles allowing to hit the target, a least squares estimation has been performed, using a set of fixed nominal values of the remaining state variables, i.e. only $\theta_{0}, \psi_{0}$ were estimated. The resulting values have been selected as the new nominal values for GSA. An uncertainty interval of $\pm 0.00049 \mathrm{rad}$ has been assumed on $\theta_{0}$ and $\psi_{0}$ that corresponds to the measurement precision of the launcher's elevation and azimuth at the ISL's test range. The initial roll angle $\phi_{0}$ can be fixed to zero, as it does not influence the trajectory.

All the nominal values of state variables are summarized in Table 1, together with their imposed uncertainty intervals. 
Table 1 Uncertain initial state variables

\begin{tabular}{lcccccc}
\hline & Nominal value & & & & & \\
\cline { 2 - 5 } State & Scenario A & Scenario B & Scenario C & Scenario D & Uncertainty & Unit \\
\hline$u_{0}$ & 300 & 400 & 500 & 600 & $\pm 1 \%$ & $\mathrm{~m} \mathrm{~s}^{-1}$ \\
$q_{0}$ & 0 & 0 & 0 & 0 & \pm 3 & $\mathrm{rad} \mathrm{s}^{-1}$ \\
$r_{0}$ & 0 & 0 & 0 & 0 & \pm 3 & $\mathrm{rad} \mathrm{s}^{-1}$ \\
$\theta_{0}$ & 0.0602 & 0.0370 & 0.0251 & 0.0188 & \pm 0.00049 & $\mathrm{rad}$ \\
$\psi_{0}$ & -0.0009 & -0.0008 & -0.0006 & -0.0005 & \pm 0.00049 & $\mathrm{rad}$ \\
\hline
\end{tabular}

\section{Uncertainties of model parameters}

In the context of free-flight experiments performed at ISL, the in-flight behavior of a projectile is investigated for a flat-fire scenario. Under such circumstances, the axial force coefficient $C_{X}$ and normal force coefficient $C_{N_{\alpha}}$ have a high influence on projectile dynamics [6]. The pitching moment is considered as well-known for the $155 \mathrm{~mm}$ architecture. Consequently, for this study only $C_{X}$ and $C_{N_{\alpha}}$ have been chosen as the objects of interest for GSA. The nominal values of all aerodynamic coefficients are known in a form of lookup tables, for which an initial guess is based on previous free-flight experiments, results from the literature, semi-empirical codes, CFD simulations, etc. Each of the methods offers a different way to quantify the projectile aerodynamics, hence it can also be considered as a source of uncertainty over the initial guess of the values of the aerodynamic coefficients. Herein, an additional 5\% of uncertainty is considered. Secondly, an uncertainty of the projectile's mass $m$ is imposed. The choice of mass as a parameter of interest is on one hand realistic, since some mass uncertainty is to be expected [6]; and on the other hand, mass uncertainty has only a negligible influence on the trajectory over a short-range firing. Therefore, it can be considered as an example of parameter which would be difficult to identify using embedded sensors.

All the uncertainty intervals of the uncertain model parameters are summarized in Table 2

Table 2 Uncertain projectile parameters

\begin{tabular}{lccc}
\hline Parameter & Nominal value & Uncertainty & Unit \\
\hline$m$ & 45 & $\pm 0.25 \%$ & $\mathrm{~kg}$ \\
$C_{X}$ & tabular & $\pm 5 \%$ & - \\
$C_{N_{\alpha}}$ & tabular & $\pm 5 \%$ & - \\
\hline
\end{tabular}

An underlying assumption permitting the use of variance-based techniques lays in the modality of the uncertainty distribution. Shall the distribution be, e.g. multi-modal, other techniques—such as entropy-based approach—would be preferred [39]. An another assumption is the one of a uniform distribution of the uncertainty of model parameters. Even though such an assumption might in some cases change the results of sensitivity analysis (e.g. in [40]), it is chosen here both for the sake of simplicity and due to the lack of parameter values around which the distribution could be easily centered, especially for the case of aerodynamic coefficients. Should such a value exist, normal or triangular 
distributions may become a better choice. In the present case, there is no a priori reason for any parameter value to be more probable than another one in the uncertainty interval.

\section{Global Sensitivity Analysis theory}

Sensitivity analysis answers the question of how can the uncertainty of the model output be apportioned to different sources of uncertainty in the model input [7]. Such a knowledge offers the possibility of assessing the influence of parameter uncertainties on the model behavior, which can be applied for several purposes [8-10], two of which are of interest for the present article:

- Test the understanding of the model, e.g. by testing the coherency between the results and the a priori knowledge in the field, in order to confirm the physical behavior of the simulated model.

- Provide insight into identifiability of the model's parameters, i.e. taking advantage of the knowledge that non-influential parameters are also non-identifiable [41]. Therefore, GSA might be used to answer the question if the uncertainty in sensor signals can be apportioned to any of the uncertain parameters - if not, they would be deemed non-identifiable.

In the context of GSA, the general model structure from Equation (1) has to be extended to allow for stochastic variables to be present in the model. Therefore, we will consider a modified structure $\mathcal{M}_{G S A}$, such that:

$$
\mathcal{M}_{G S A}:\left\{\begin{array}{l}
\dot{\mathbf{x}}(t, \chi)=f\left(\mathbf{x}(t, \chi), \mathbf{C}\left(\mathbf{x}(t, \chi), \mathbf{p}_{a}(\chi)\right)\right), \quad \mathbf{x}(0, \chi)=\mathbf{x}_{0} \\
\mathbf{y}(t, \chi)=g(\mathbf{x}(t, \chi))
\end{array}\right.
$$

where $\mathbf{y}(t)=\left[A_{x}, A_{y}, A_{z}, G_{y}, G_{z}, H_{x}, H_{y}, H_{z}\right]^{T}$. The model 12 depends on the initial state vector $\mathbf{x}(0, \chi)$ and on the vector of parameters of aerodynamic coefficients $\mathbf{p}_{a}(\chi)$. These two vectors contain elements that are uncertain and can be considered as random variables, dependent on an elementary random event $\chi$. The vector of their uncertain elements is denoted as $\boldsymbol{\Omega} \in \mathbb{R}^{s}, \boldsymbol{\Omega}=\left[\Omega_{1}(\chi), \Omega_{2}(\chi), \ldots, \Omega_{s}(\chi)\right]$, where $\Omega_{i}(\chi) \in \mathbb{R}$. For the sake of simplicity, the notation $(\chi)$ will be dropped hereinafter.

\section{A. Sensitivity indices}

Sensitivity indices allow to quantify how much, on average, the output variance would be reduced if an uncertain parameter $\Omega_{i} \in \mathbf{\Omega}$ was fixed to its constant nominal value. In other words, they allow to assess the effect that the uncertain parameter $\Omega_{i}$ has on a $j$-th output of the model, noted as $y_{j}$. Main sensitivity indices are defined for any given time instant, following [7, 42], as:

$$
S_{i}^{j}=\frac{V\left(E\left(y_{j} \mid \Omega_{i}\right)\right)}{V\left(y_{j}\right)} .
$$

where $V\left(y_{j}\right)$ is the total variance of $y_{j}$ and $V\left(E\left(y_{j} \mid \Omega_{i}\right)\right)$ is the conditional variance of $y_{j}$ given $\Omega_{i}$. 
It is assumed that uncertain parameters $\Omega_{i}$ are independent. The value of $S_{i}$ lies between 0 and 1 . The closer to 1 , the higher the contribution of $\Omega_{i}$ to the total variance of the output. The sum of main sensitivity indices offers the following insight into the influence of uncertain parameters on the model, for a given output signal:

- $\sum_{i} S_{i}=1$ if the model is additive, indicating that the main sensitivity indices are sufficient to fully describe the model uncertainties.

- $\sum_{i} S_{i}<1$ otherwise, indicating the influence of interactions between model parameters [43].

In the latter case, total sensitivity indices can be used to assess both the individual influence of a parameter $\Omega_{i}$, and its interactions with other parameters [44]. The total sensitivity indices are defined as:

$$
S_{T_{i}}^{j}=\frac{E\left(V\left(y_{j} \mid \Omega_{\sim i}\right)\right)}{V\left(y_{j}\right)} .
$$

where notation $\Omega_{\sim i}$ indicates all the elements of $\boldsymbol{\Omega}$ except for $\Omega_{i}$.

Similarly as in the case of the main sensitivity indices, the sum of all total indices for a given output signal:

- $\sum_{i} S_{T_{i}}=1$ if the model is additive,

- $\sum_{i} S_{T_{i}}>1$ otherwise.

Overall, all the important rules for interpreting the sensitivity indices, with respect to a selected output signal, are summarized in Table 3 .

Table 3 Rules for interpreting the obtained sensitivity indices.

\begin{tabular}{|c|l|c|}
\hline Observation & Interpretation & Reference \\
\hline$S_{i} \approx 1$ & The parameter $x_{i}$ influences directly the output uncertainty. & {$[7]$} \\
$S_{i} \approx 0$ & The parameter $x_{i}$ does not influence directly the output uncertainty. & {$[7]$} \\
$\sum_{i}^{s} S_{i} \approx 1$ & The model is additive, interactions are not present, i.e. $S_{T_{i}}=S_{i}$ & {$[43]$} \\
$S_{T_{i}} \approx 1$ & The parameter $x_{i}$ influences (directly or via interactions) the output & {$[7]$} \\
$S_{T_{i}} \approx 0$ & uncertainty. & The parameter $x_{i}$ does not influence the output uncertainty and is \\
& non-identifiable. & {$[7,45]$} \\
\hline
\end{tabular}

\section{B. Estimation of the sensitivity indices}

Since the original Sobol' work from 1993, several methods have been developed for computing the variance-based sensitivity indices in more computationally efficient way, such as: the Fourier amplitude sensitivity test [46, 47], the surrogate models approach [48,-50], and Monte Carlo simulation improved with various techniques to reduce its computational complexity [9, 51, 52]. Therefore, the choice of sensitivity indices estimation method is not a trivial task.

In this paper, the approach of applying GSA, as opposed to the local analysis, is proposed. For this purpose, the Monte Carlo simulation-based method is suggested, as it allows a direct computation of sensitivity indices, and for its 
simplicity in implementation. The authors take advantage of the non-rolling frame transformation in order to reduce the simulation time of each model run.

In order to reduce the required number of simulations, a permutation-based approach proposed by Mara [51] is employed. This approach consists of three steps:

1) A first model response vector $Y^{1}=g\left(\Omega^{1}\right)$ is generated, based on the measurements function $g$ of the first Sobol' sample $\Omega^{1}$. The sample matrix has a size $N \times s$, where $N$ is the amount of Monte-Carlo samples, and $s$ is the number of uncertain model parameters.

2) A second sample matrix $\Omega^{2}$ is generated based on column-wise permutation of $\Omega^{1}$ matrix: each $i$-th column (out of $d$ columns in total) is permuted by a permutation vector $r_{i}$. Hence, noting the permutation matrix as $R=\left[r_{1}, r_{2}, \ldots, r_{s}\right]$, the second sample matrix is obtained as $\Omega^{2}=\Omega^{1} \circ R$. The second model response vector is calculated as $Y^{2}=g\left(\Omega^{2}\right)$.

3) Main sensitivity indices are calculated for each of $j$ output signal with respect to each of $s$ uncertain parameters, as:

$$
\hat{S}_{i}^{j}=\frac{(N-1)^{-1} \sum_{k=1}^{N}\left(y_{k j}^{r_{i}}-\hat{\mu}_{1}^{j}\right)\left(y_{k j}^{2}-\hat{\mu}_{2}^{j}\right)}{\hat{\sigma}_{1}^{j} \hat{\sigma}_{2}^{j}},
$$

where $y_{k j}^{r_{i}}$ is k-th element of the $\mathrm{j}$-th output signal obtained through reordering the $y_{k j}^{1}$ accordingly with column-wise permutation $r_{i}$. Symbols $\mu$ and $\sigma$ denote the mean value and the standard deviation, respectively, and . indicates an estimated value.

For the purpose of upcoming GSA, eight parameters from Tables 1 and 2 have been deemed as uncertain: $\Omega=\left[u_{0}, q_{0}, r_{0}, \theta_{0}, \psi_{0}, m, C_{X}, C_{N_{\alpha}}\right]^{T}$. Such a setting of uncertain parameters allows to evaluate their influence on the system dynamics, which in turn influences the sensors' measurements, as noted in Fig. 1 Therefore, GSA can be used to answer which parameters, and to what extent, do influence the measurement sensor equations. Moreover, the number of uncertain parameters is equal to $s=8$, whereas each simulation of a single trajectory of the quasi-LPV model lasts less than three seconds [28]. Such a case further supports the selection of a variance-based methods as the basis of the global sensitivity assessment: for the cases when the number of parameters is lower than 20 , and the CPU time per one run is lower than one minute, the choice of variance-based methods is suggested as a rule-of-a-thumb by De Rocquingy [13], as well as by Saltelli et al. [8].

\section{Simulation results}

Simulations for the purpose of GSA using Mara's method have been performed based on the set of nominal conditions and their uncertainty intervals as summarized in Tables 1 and 2 The analysis has been performed in a time-varying approach —as information on sensitivity could be hidden at some periods of time, while being readily visible in the others [53]. The value of sensitivity indices is calculated every $2 \mathrm{~ms}$, which is faster than the quasi-LPV 


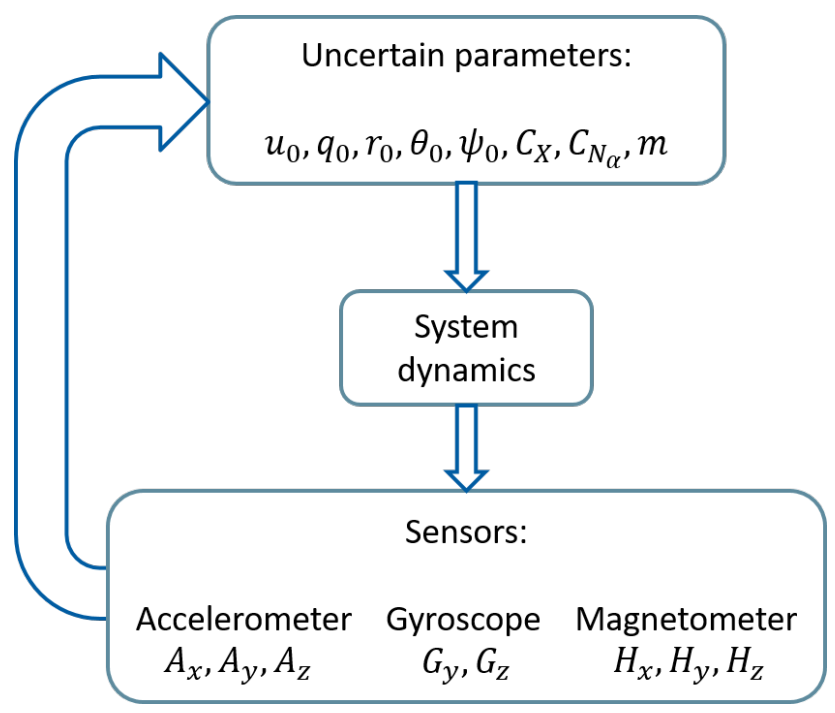

Fig. 1 Diagram of the global sensitivity analysis.

model dynamics. Thus, an accurate representation of the time-varying behavior of the model is possible.

The procedure of obtaining the results can be summarized as follows:

- Perform a sampling of the uncertain parameter space, using the uncertainty ranges as dictated by Tables 1 and 2 , while assuming that the samples are uniformly distributed. The sampling size, for which convergent results have been obtained, equals to two samples $\Omega^{1}$ and $\Omega^{2}$, each of the same size, with the total sample size of $N=40000$.

- Simulate the model trajectory (i.e. the values of the time-varying output signals) for each of the $N$ samples over the distance of $1 \mathrm{~km}$ corresponding to the distance between the projectile launcher and the sandbay.

- Calculate the sensitivity indices $S_{i}, i=1,2, \ldots, s$, based on Equation $[15$ for every time instant.

\section{A. Results interpretation for the full parameter vector}

All the main sensitivity indices, calculated using Mara's method, have been plotted for each time instant (see Figure 2 and 3 .

Firstly, the initial translational and angular velocities are dominating the uncertainty of the output signals, as shown in Fig. 2 (a) for the example of the radial accelerometer $A_{y}$. This result, although in-line with physical expectations, obscures the influence of other parameters on the output signals. That is especially visible for the case of the aerodynamic coefficients. For instance, as shown in Fig. 2 (b), it appears as if uncertainty around the nominal value of $C_{X}$ does not visibly influence the measurement signals other than the axial accelerometer $A_{x}$. Such an effect would be worrying, as it could indicate non-identifiability of the aerodynamic coefficients by most of the sensors. However, this effect stems from the exponentially higher influence of initial velocities compared to other factors, rather than from the suspected lack of influence of the aerodynamic coefficients. Since $u_{0}, q_{0}, r_{0}$ almost totally dominate, only a little effect is visible from the other parameters as observed through sensitivity indices normalised by definition to a $[0,1]$ range. Sensitivity 
indices are relative to each other, and hence neither can they be directly compared between outputs, nor will a clear influence of the uncertain parameter be visible if one of them logarithmically dominates. The uncertain translational and angular velocities influence the sensors uncertainty to such an extent, that the desired effects of Euler angles and aerodynamic coefficients uncertainty is invisible. The oscillations visible in in Fig. 2 (a) stem from the rotation of a spin-stabilised projectile, and change over time together with changing precession/nutation patterns typical for spin-stabilised projectiles [30].

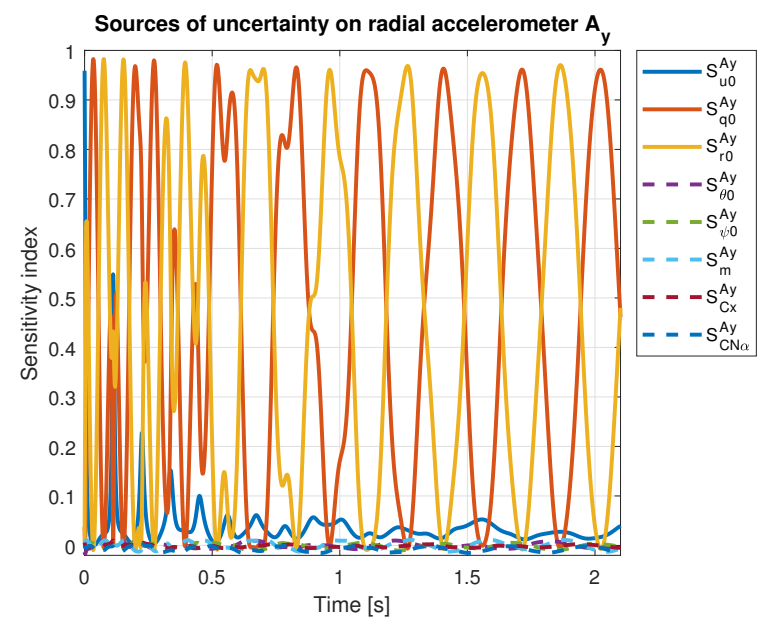

(a) Sensitivity indices of each uncertain parameter with respect to $A_{y}$. Influence of translational and angular velocities dominates over all the other parameters.

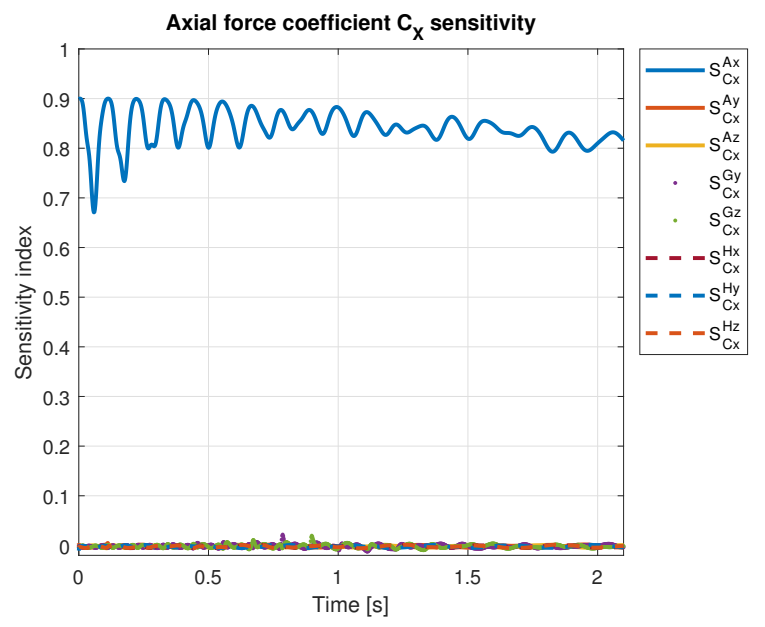

(b) Main sensitivity indices of the output sensors with respect to $C_{X}$. The influence of model uncertainties on sensors other than the axial accelerometer $A_{x}$ is visibly low.

Fig. 2 Exemplary results: a proof of the domination of angular and translational velocities over all the other uncertain parameters.

Another explanation would be to conclude that the influence of uncertain parameters is expressed through their interactions, and thus the total sensitivity indices would have to be calculated. However, such an assumption can be easily disproved, since $\sum_{i=1}^{s} S_{i}^{j}>0.9$ for almost all of the outputs $j$ for the whole duration of the flight, which in accordance with Table 3 implies that the model is approximately additive (Fig. 3 . Thus, the main indices $S_{i}^{j}$ are equal to the total sensitivity indices $S_{T_{i}}^{j}$.

In order to be able to assess the influence of the uncertainty of the aerodynamic coefficients, as well as of the other less influential model parameters, it has been decided to repeat the analysis for a modified set of uncertain parameters, as explained below.

\section{B. Results interpretation for the reduced parameter vector}

In the sequel, a modified set of parameters is examined. This time, the initial velocities are considered only at their nominal values, hence $\Omega=\left[\theta_{0}, \psi_{0}, m, C_{X}, C_{N_{\alpha}}\right]^{T},(s=5)$.

The results observed in such a setting, as shown in Fig. 4 to 6 are more promising, as it is now visible that they 


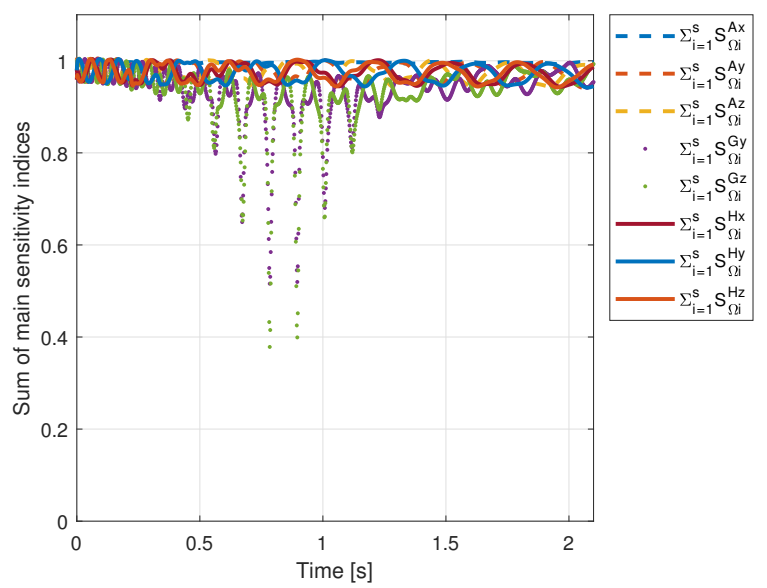

Fig. 3 Sum of main sensitivity indices for each output signal. The results suggest the additive nature of the model.

confirm the predictions of the domain knowledge of ballistics and aerodynamics:

- The initial Euler angles $\theta_{0}$ and $\psi_{0}$ influence only the magnetometer measurements $\left(H_{x}, H_{z}\right.$ and $H_{y}, H_{z}$ respectively, due to the angles acting in $x z$ and $y z$ planes), and their influence quickly diminishes over the time of flight. It is visible in Fig. 4 (a) and (b), on the example of magnetometer sensitivities (dotted line) and accelerometer sensitivities (solid lines): influence of Euler angles uncertainty on accelerometers is close to zero, suggesting their non-identifiability. In contrast, uncertainty of Euler angles explains most of magnetometers uncertainty initially, but the influence decreases to almost zero over just two seconds of flight.

- The effect of the uncertainty on axial force coefficient $C_{X}$ is visible especially on $A_{x}$ measurements: in Fig. 5 (a), its sensitivity (solid blue line) remains close to one during the whole flight. Moreover, radial accelerometers $A_{y}, A_{z}$ (solid red and yellow lines) are influenced by $C_{X}$ in the time instances, when they are not already strongly influenced by $C_{N_{\alpha}}$. Finally, by comparing Fig. 4(b) and Fig. 5(a), it can be concluded that the influence of Euler angles on magnetometers $H_{x}, H_{y}, H_{z}$ is visible in the time instances when it is not dominated by $C_{X}$ (dotted lines at both Figures).

- The effect of normal force coefficient $C_{N_{\alpha}}$ uncertainty is visible in the radial accelerometer measurements, but not in the axial one. It can be seen in Fig. 5(b) that the axial accelerometer sensitivity $S_{C_{N_{\alpha}}}^{A_{x}}$ (solid blue line) remains close to zero. Such a result also suggests its non-identifiability when only axial accelerometer $A_{x}$ data would be used. Radial accelerometer sensitivities $S_{C_{N_{\alpha}}}^{A_{y}}, S_{C_{N_{\alpha}}}^{A_{z}}$ (red and yellow solid lines) significantly better explain $C_{N_{\alpha}}$ uncertainty.

- The mass influence on sensor measurements are negligible. This effect is visible in Fig. 6)(a), since all sensitivities remain close to zero. Such a result also suggests that mass would be effectively impossible to identify using only the embedded sensors. 


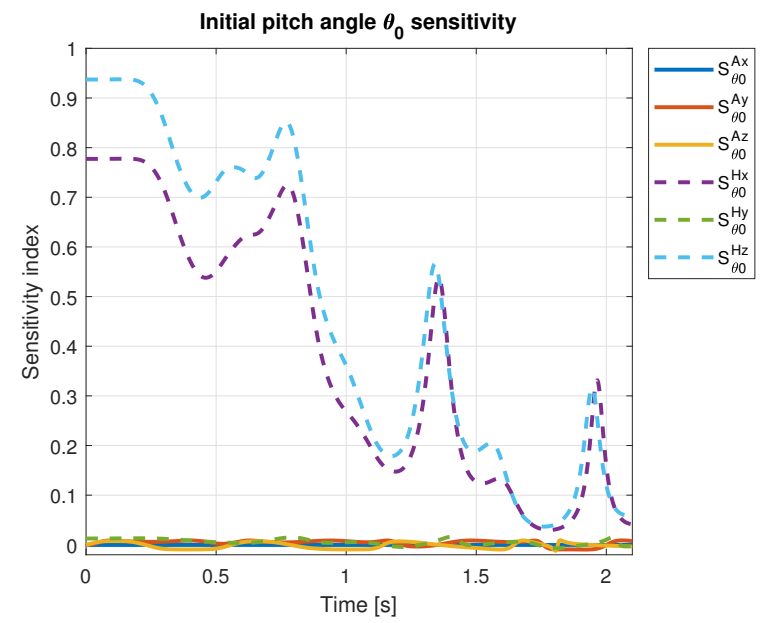

(a) The uncertainty of $\theta_{0}$ influences only the magnetometer measurements $H_{x}, H_{z}$, especially in the beginning. Any attempt to identify it using accelerometers would be futile.

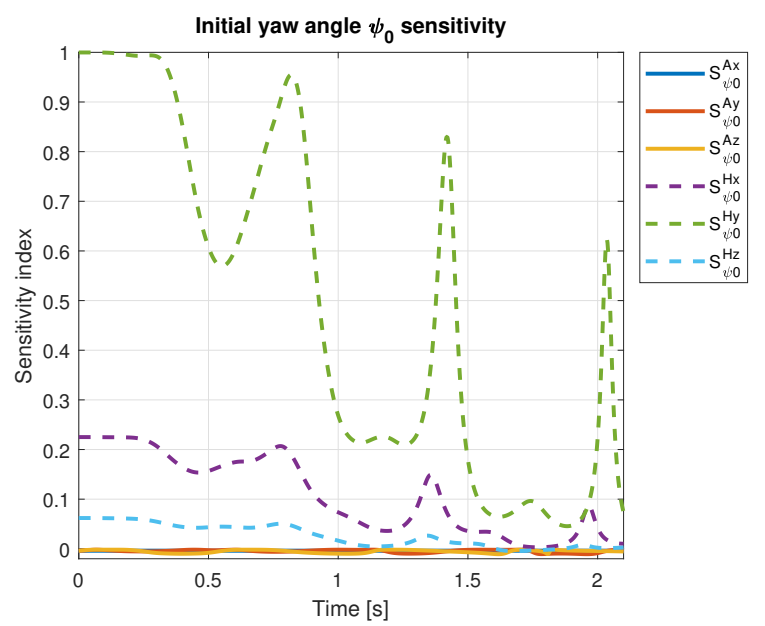

(b) The uncertainty of $\phi_{0}$ influences the magnetometer measurements $H_{x}, H_{y}$, especially in the beginning. Any attempt to identify it using accelerometers would be futile.

Fig. 4 Sensitivity indices with respect to the initial Euler angles.

The observed effects are especially interesting in the context of identification of unknown parameters. Knowing that non-influential parameters are non-identifiable [41], one can easily confirm that some of the model parameters might be identifiable using only some of the sensors, and only in some periods of time. For example, any attempt to identify initial Euler angles $\theta_{0}$ and $\psi_{0}$ using magnetometer measurements $H_{x}, H_{y}, H_{z}$ after a few seconds of flight would be futile. Moreover, the conformity of the results with the domain knowledge combined with the previously described numerical accuracy of the quasi-LPV model [28], suggests that the quasi-LPV model has been correctly developed, and effectively preserves the real behavior of a projectile. It can be also concluded that the model with reduced uncertain parameter vector becomes more additive, as can be seen in Fig. 6(b) in comparison with Fig. 3. Additive nature of the model depends heavily on the selection of uncertain parameters and different selection might have resulted in a non-additive structure - in such case, a separate calculation of total sensitivity indices would have been necessary, e.g. using standard Monte Carlo approach [54]. It is worth noting that the correctness of the obtained sensitivity indices evolution can be additionally tested by calculating the sensitivity indices for denser time-grids. For the studied model, results remain equivalent to those obtained for a lower density of time instances.

Overall tendencies of the sensitivity indices have been summarised in Tables 4 and 5

It may be noted that convergence of sensitivity indices estimates is assured through the use of sufficiently large number of samples; that in turn is rendered possible through the use of computationally efficient quasi-LPV model developed in a non-rolling reference frame. Should the model complexity be higher and calculations prohibitively computationally expensive, an approach of calculating confidence intervals around the estimated could be utilised. An example of such a technique is presented in [55]. Alternatively, different sampling technique could be applied, as it can 


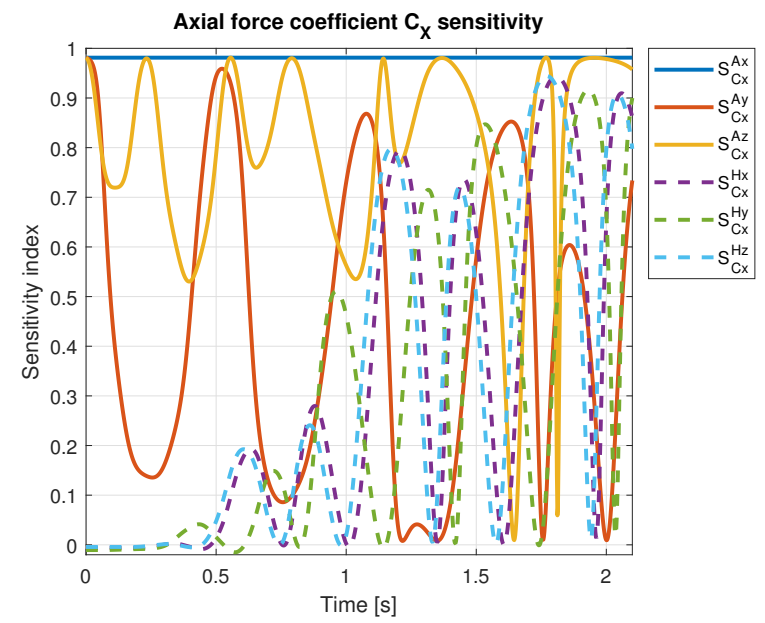

(a) $C_{X}$ influences especially the axial accelerometer $A_{x}$, as well as the radial measurements $A_{y}, A_{z}$ in the instances of time when they are not influenced by $C_{N \alpha}$.

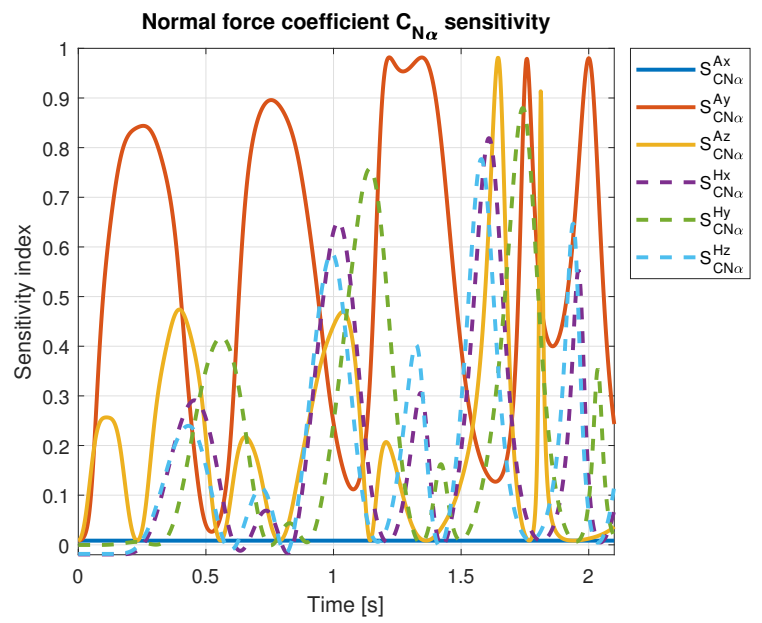

(b) $C_{N \alpha}$ influences especially the radial accelerometer $A_{y}, A_{z}$, with an increasing over time influence on the magnetometers $H_{x}, H_{y}, H_{z}$.

Fig. 5 Sensitivity indices with respect to the aerodynamic coefficients.

lead to a better coverage of the parameter space and can accelerate the convergence of the sensitivity indices, without requiring a large number of samples [7].

Table 4 Sensitivity of uncertain state variables.

\begin{tabular}{ccccccccc} 
& $A_{x}$ & $A_{y}$ & $A_{z}$ & $H_{x}$ & $H_{y}$ & $H_{z}$ & $G_{y}$ & $G_{z}$ \\
\hline$\theta_{0}$ & ni & ni & ni & ds & ni & ds & ni & ni \\
$\psi_{0}$ & ni & ni & ni & ds & ds & ni & ni & ni \\
$m$ & ni & ni & ni & ni & ni & ni & ni & ni \\
\hline
\end{tabular}

Legend: ni - parameter non-influential and non-identifiable, ds - sensitivity decreasing over time.

\section{Conclusions}

In the paper, a quasi-LPV reformulation of a nonlinear first-principles-based model of a projectile in free-flight is described, on top of which a global sensitivity analysis is applied (GSA). The suggested approach aims at using the proposed quasi-LPV model—which is much faster due to the applied non-rolling reference frame-rather than the nonlinear one, in order to shorten the duration of each simulation. For GSA, a permutation-based sampling is used, in order to reduce the total number of simulations.

Benefits of GSA are twofold. Firstly, it highlights the possible identifiability limitations. Since non-sensitive parameters are non-identifiable, the approach has allowed to determine which parameters of the model can be deemed non-identifiable, either during the whole flight (i.e. mass of the projectile), or during some periods of it (e.g. initial Euler angles after the first seconds of flight). This aspect of work can be considered as a practical approach to gain more insight into the parametric variations within the model. Secondly, in the context of the present work, GSA is considered 


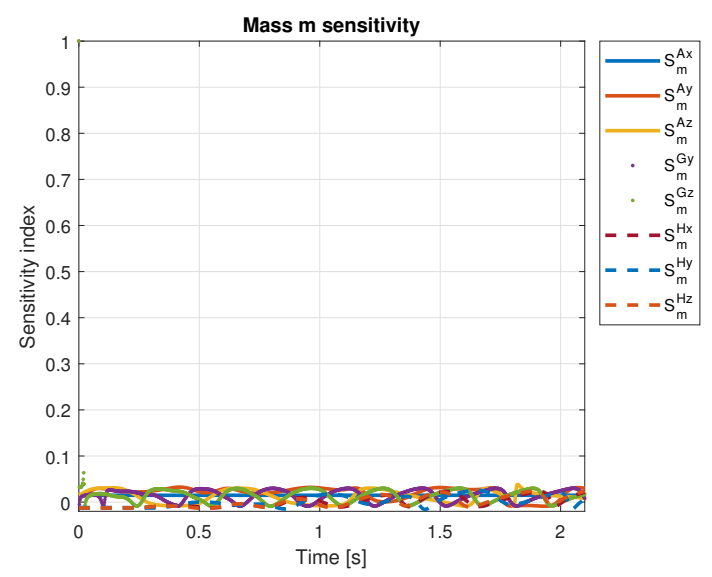

(a) Main sensitivity indices of the output sensors with respect to $m$. Mass uncertainty influences the sensors only slightly. An attempt to identify it using in-flight measurements would be difficult.

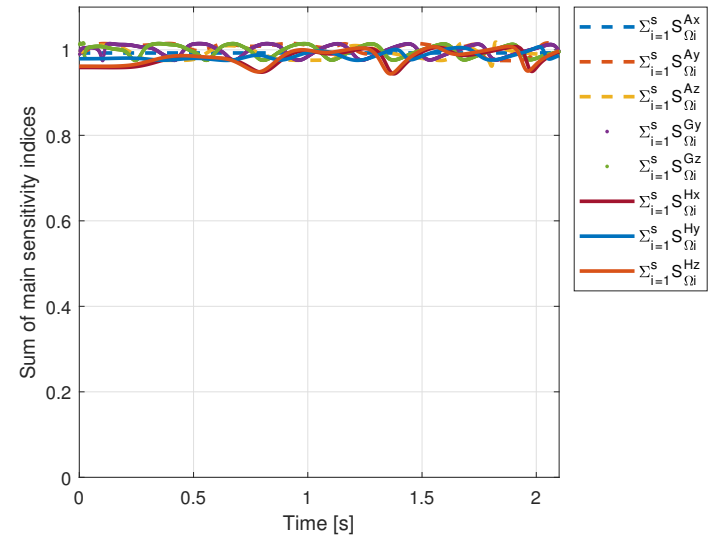

(b) Sum of main sensitivity indices. Results are approximately equal to one, indicating additive nature of the model.

Fig. 6 Exemplary results: a proof of the additive nature of the model and non-identifiability of the mass.

Table 5 Sensitivity of uncertain model parameters.

\begin{tabular}{ccc} 
& $C_{X}$ & $C_{N_{\alpha}}$ \\
\hline$A_{x}$ & dominant & non-identifiable \\
$A_{y}$ & sinusoidal decrease & sinusoidal increase \\
$A_{z}$ & dominates, oscillatory & sinusoidal increase \\
$H_{x}$ & average, oscillatory & sinusoidal increase \\
$H_{y}$ & average, oscillatory & sinusoidal increase \\
$H_{z}$ & average, oscillatory & sinusoidal increase \\
$G_{y}$ & dominates, oscillatory & dominates, oscillatory \\
$G_{z}$ & dominates, oscillatory & dominates, oscillatory \\
\hline
\end{tabular}

as a tool to analyse the behavior of the model. That, in turn, allows to confirm that the quasi-LPV model—which has previously been tested only for its numerical precision — correctly represents the expected physical behavior of a studied system.

The future work will concentrate on applying GSA as a standardized tool for performing pre-experimentation procedure of different projectile architectures in the context of free-flight experiments conducted at the French-German Research Institute of Saint-Louis. As such, it will be used in tandem with uncertainty analysis that aim to evaluate the maximum dispersion of the impact points of the projectiles.

\section{Funding Sources}

This work is in part financed by the French Defense Procurement Agency (DGA, Direction Générale de l'Armement) of the French Ministry of the Armed Forces. 


\section{References}

[1] Zipfel, P. H., Modeling and Simulation of Aerospace Vehicle Dynamics, $2^{\text {nd }}$ ed., AIAA Inc., Blacksburg VA, 2007.

[2] Hamel, N., and Gagnon, E., "CFD and Parametric Study on a $155 \mathrm{~mm}$ Artillery Shell Equipped with a Roll-Decoupled Course Correction Fuze," 29th AIAA Applied Aerodynamics Conference, 2011, pp. 1-10.

[3] Bryson, J., Vasile, J. D., Celmins, I., and Fresconi, F., “Approach for Understanding Range Extension of Gliding Indirect Fire Munitions," 2018 AIAA Atmospheric Flight Mechanics Conference, 2018, pp. 1-20. doi:10.2514/6.2018-3158.

[4] Strub, G., "Modeling, Identification and Control of a Guided Projectile in a Wind Tunnel," Ph.D. thesis, Universite de Haute-Alsace, 2016.

[5] Albisser, M., Dobre, S., Berner, C., Thomassin, M., and Garnier, H., “Aerodynamic Coefficient Identification of a Space Vehicle from Multiple Free-Flight Tests,” Journal of Spacecraft and Rockets, Vol. 54, No. 2, 2017, pp. 426-435. doi:10.2514/1.A33587.

[6] Carlucci, D. E., and Jacobson, S. S., Ballistics: Theory and Design of Guns and Ammunition, $3^{\text {rd }}$ ed., Taylor \& Francis Group, Boca Raton, Miami, Florida, USA, 2018.

[7] Saltelli, A., Ratto, M., Andres, T., Campolongo, F., Cariboni, J., Gatelli, D., Saisana, M., and Tarantola, S., Global Sensitivity Analysis. The Primer, Wiley and Sons, 2007. doi:10.1002/9780470725184.

[8] Saltelli, A., Tarantola, S., Campolongo, F., and Ratto, M., Sensitivity analysis in practice, John Wiley \& Sons Ltd., 2004.

[9] Iooss, B., and Lemaître, P., “A Review on Global Sensitivity Analysis Methods,” Uncertainty Management in SimulationOptimization of Complex Systems, Vol. 59, edited by G. Dellino and C. Meloni, Springer US, 2015, Chap. 5, pp. 101-122. doi:10.1007/978-1-4899-7547-8.

[10] Cukier, R. I., Levine, H. B., and Shuler, K. E., "Nonlinear Sensitivity Analysis of Multiparameter Model Systems," Journal of Computational Physics, Vol. 26, No. 1, 1978, pp. 1-42.

[11] Hamby, D. M., “A review of techniques for parameter sensitivity analysis of environmental models,” Environmental Monitoring and Assessment, Vol. 32, No. 2, 1994, pp. 135-154. doi:10.1007/BF00547132.

[12] Sobol, I. M., "Sensitivity estimates for nonlinear mathematical models," Mathematical modelllng and computational experiments, Vol. 1, No. 4, 1993, pp. 407-414.

[13] de Rocquigny, E., Devictor, N., and Tarantola, S., "Chapter 18. Sensitivity analysis methods," Uncertainty in Industrial Practice, Wiley and Sons, 2008, pp. 259-284.

[14] Torres, S., “Trajectory Accuracy Sensitivity to Modeling Factors," 15th AIAA Aviation Technology, Integration, and Operations Conference AIAA, Dallas, TX, 2015, pp. 1-19. doi:10.2514/6.2015-2599.

[15] Wang, L., Diskin, B., Biedron, R., Nielsen, E. J., and Bauchau, O., "Sensitivity Analysis of Multidisciplinary Rotorcraft Simulations," AIAA Scitech 2017 Forum, 2017, pp. 1-14. doi:10.2514/6.2017-1670. 
[16] York, M. A., Öztürk, B., Burnell, E., and Hoburg, W. W., "Efficient Aircraft Multidisciplinary Design Optimization and Sensitivity Analysis via Signomial Programming,” AIAA Journal, Vol. 56, No. 11, 2018, pp. 4546-4561. doi:10.2514/1.j057020.

[17] Dannenhoffer, J., and Haimes, R., "Design Sensitivity Calculations Directly on CAD-based Geometry," AIAA Scitech 2015 Forum, 2015, pp. 1-25. doi:10.2514/6.2015-1370.

[18] Makhija, D., and Beran, P. S., "Spiral: A General Framework For Parameter Sensitivity Analysis," AIAA Scitech 2017 Forum, 2017, pp. 1-16. doi:10.2514/6.2017-1306.

[19] Albisser, M., and Dobre, S., "Sensitivity Analysis for Global Parameter Identification. Application to Aerodynamic Coefficients," IFAC-PapersOnLine, Vol. 51, No. 15, 2018, pp. 963-968. doi:10.1016/j.ifacol.2018.09.069.

[20] Huan, X., Safta, C., Sargsyan, K., Geraci, G., Eldred, M. S., Vane, Z. P., Lacaze, G., Oefelein, J. C., and Najm, H. N., “Global Sensitivity Analysis and Estimation of Model Error, Toward Uncertainty Quantification in Scramjet Computations," AIAA Journal, Vol. 56, No. 3, 2018, pp. 1170-1184. doi:10.2514/1.J056278.

[21] Wei, P., Lu, Z., and Song, J., "Extended Monte Carlo Simulation for Parametric Global Sensitivity Analysis and Optimization," AIAA Journal, Vol. 52, No. 4, 2014, pp. 867-878. doi:10.2514/1.j052726.

[22] Jiang, Z., Chen, W., and German, B. J., "Multidisciplinary Statistical Sensitivity Analysis Considering Both Aleatory and Epistemic Uncertainties,” AIAA Journal, Vol. 54, No. 4, 2016, pp. 1326-1338. doi:10.2514/1.J054464.

[23] Hale, L. E., Patil, M., and Roy, C. J., "Sensitivity Analysis Methods for Systems with Epistemic Uncertainties," AIAA Scitech 2015 Forum, 2015, pp. 1-8. doi:10.2514/6.2015-1818.

[24] Rogers, J., Costello, M., Harkins, T., and Hamaoui, M., "Effective use of magnetometer feedback for smart projectile applications," Navigation, Journal of the Institute of Navigation, Vol. 58, No. 3, 2011, pp. 203-219. doi:10.1002/j.2161-4296.2011.tb02581.x.

[25] Rogers, J., and Costello, M., "A low-cost orientation estimator for smart projectiles using magnetometers and thermopiles," Navigation, Journal of the Institute of Navigation, Vol. 59, No. 1, 2012, pp. 9-24. doi:10.1002/navi.5.

[26] Sahu, J., and Fresconi, F., “Aeromechanics and Control of Projectile Roll Using Coupled Simulation Techniques,” Journal of Spacecraft and Rockets, Vol. 52, No. 3, 2015, pp. 944-957. doi:10.2514/1.A33133, URL http://arc.aiaa.org/doi/10. 2514/1.A33133

[27] Machala, D., Dobre, S., Albisser, M., Collin, F., and Gilson, M., "Quasi-LPV modelling of a projectile's behaviour in flight,” 18th IFAC Symposium on System Identification, SYSID2018, Elsevier B.V., 2018, pp. 1080-1085. doi:10.1016/j.ifacol.2018.09.050.

[28] Machala, D., Dobre, S., Albisser, M., Gilson, M., and Collin, F., “The Analysis of Vehicle's In-Flight Behaviour Using Quasi-LPV and Nonlinear Models,” AIAA Scitech 2019 Forum, San Diego, CA, 2019, pp. 1-12. doi:10.2514/6.2019-1321.

[29] Albisser, M., "Identification of aerodynamic coefficients from free flight data," Ph.D. thesis, University of Lorraine, 2015. 
[30] Wernert, P., Theodoulis, S., and Morel, Y., "Flight Dynamics Properties of $155 \mathrm{~mm}$ Spin-Stabilized Projectiles Analyzed in Different Body Frames," AIAA AFM Conference, 2010, pp. 1-17. doi:10.2514/6.2010-7640.

[31] Barnett, B., “Trajectory equations for a six-degree-of-freedom missile,” Tech. rep., Picatinny Arsenal, Dover, New Jersey, 1962.

[32] Pfifer, H., "Quasi-LPV Model of a NDI-Controlled Missile Based on Function Substitution,” AIAA GNC Conference, 2012, pp. 1-12. doi:10.2514/6.2012-4970.

[33] Kwiatkowski, A., and Werner, H., "Parameter Reduction for LPV Systems via Principal Components Analysis," IFAC Proceedings Volumes, Vol. 38, No. 1, 2005, pp. 596-601. doi:10.3182/20050703-6-CZ-1902.00100.

[34] Sève, F., Theodoulis, S., Wernert, P., Zasadzinski, M., and Boutayeb, M., "Pitch/Yaw Channels Control Design for a 155mm Projectile with Rotating Canards, using a H-infinity Loop-Shaping Design Procedure," AIAA GNC Conference, 2014, pp. 1-24. doi:10.2514/6.2014-1474.

[35] Changey, S., Pecheur, E., and Brunner, T., “Attitude Estimation of a projectile using Magnetometers and Accelerometers: Experimental Validation,” IEEE/ION PLANS Symposium 2014, 2014, pp. 1168-1173. doi:10.1109/PLANS.2014.6851488.

[36] Albisser, M., Dobre, S., Decrocq, C., Saada, F., Martinez, B., and Gnemmi, P., "Aerodynamic Characterization of a New Concept of Long Range Projectiles from Free Flight Data," 30th International Symposium on Ballistics, edited by S. Chocron and J. Walker, Long Beach, CA, 2017, pp. 1-12.

[37] Machala, D., "Free-Flight Projectile Behaviour: LPV modelling and global sensitivity analysis," Ph.D. thesis, Université de Lorraine, 2019.

[38] Adam, R., Bernard, L., Bieber, E., Krueger, D., Pecheur, E., Saada, F., Schmoltzi, D., Schneider, A., Thomas, C., and Changey, S., "Development of a gun-hardened IMU based on low-cost sensors: Hardware design and modeling," Tech. rep., French-German Research Institute of Saint-Louis, Saint-Louis, France, 2016.

[39] Auder, B., and Iooss, B., "Global sensitivity analysis based on entropy," Safety, reliability and risk analysis : theory, methods and applications, Taylor \& Francis Group, London, 2009, pp. 2107-2115.

[40] Paleari, L., and Confalonieri, R., "Sensitivity analysis of a sensitivity analysis: We are likely overlooking the impact of distributional assumptions," Ecological Modelling, Vol. 340, 2016, pp. 57-63. doi:10.1016/j.ecolmodel.2016.09.008, URL http://dx.doi.org/10.1016/j.ecolmodel.2016.09.008

[41] Dobre, S., Bastogne, T., Profeta, C., Barberi-Heyob, M., and Richard, A., "Limits of variance-based sensitivity analysis for non-identifiability testing in high dimensional dynamic models," Automatica, Vol. 48, No. 11, 2012, pp. $2740-2749$. doi:10.1016/j.automatica.2012.05.004.

[42] Anstett-Collin, F., Goffart, J., Mara, T. A., and Denis-Vidal, L., "Sensitivity analysis of complex models: Coping with dynamic and static inputs," Reliability Engineering and System Safety, Vol. 134, 2015, pp. 268-275. doi:10.1016/j.ress.2014.08.010. 
[43] Mara, T. A., and Tarantola, S., "Variance-based sensitivity indices for models with dependent inputs," Reliability Engineering and System Safety, Vol. 107, 2012, pp. 115-121. doi:10.1016/j.ress.2011.08.008.

[44] Homma, T., and Saltelli, A., "Importance measures in global sensitivity analysis of nonlinear models," Reliability Engineering \& System Safety, Vol. 52, 1996, pp. 1-17. doi:10.1016/0951-8320(96)00002-6.

[45] Dobre, S., Berner, C., Albisser, M., and Saada, F., "MarcoPolo-R ERC Dynamic Stability Experimental Campaign ISL Open Range Free Flight Tests,” Tech. rep., French-German Research Institute of Saint-Louis, Saint-Louis, France, 2015.

[46] Xu, C., and Gertner, G. Z., "Uncertainty and sensitivity analysis for models with correlated parameters,” Reliability Engineering and System Safety, Vol. 93, No. 10, 2008, pp. 1563-1573. doi:10.1016/j.ress.2007.06.003.

[47] Tarantola, S., and Mara, T. A., "Variance-Based Sensitivity Indices of Computer Models With Dependent Inputs: the Fourier Amplitude Sensitivity Test,” International Journal for Uncertainty Quantification, Vol. 7, No. 6, 2017, pp. 511-523. doi:10.1615/int.j.uncertaintyquantification.2017020291.

[48] Ratto, M., Pagano, A., and Young, P. C., "Non-parametric estimation of conditional moments for sensitivity analysis," Reliability Engineering and System Safety, Vol. 94, 2009, pp. 237-243. doi:10.1016/j.ress.2008.02.023.

[49] Blatman, G., and Sudret, B., "Efficient computation of global sensitivity indices using sparse polynomial chaos expansions," Reliability Engineering and System Safety, Vol. 95, No. 11, 2010, pp. 1216-1229. doi:10.1016/j.ress.2010.06.015.

[50] Sudret, B., "Global sensitivity analysis using polynomial chaos expansions," Reliability Engineering and System Safety, Vol. 93, No. 7, 2008, pp. 964-979. doi:10.1016/j.ress.2007.04.002.

[51] Mara, T. A., and Rakoto, O., "Comparison of some efficient methods to evaluate the main effect of computer model factors," Journal of Statistical Computation and Simulation, Vol. 78, No. 2, 2008, pp. 167-178. doi:10.1080/10629360600964454.

[52] Saltelli, A., Annoni, P., Azzini, I., Campolongo, F., Ratto, M., and Tarantola, S., "Variance based sensitivity analysis of model output. Design and estimator for the total sensitivity index," Computer Physics Communications, Vol. 181, No. 2, 2010 , pp. 259-270. doi:10.1016/j.cpc.2009.09.018.

[53] Pianosi, F., and Wagener, T., "Understanding the time-varying importance of different uncertainty sources in hydrological modelling using global sensitivity analysis," Hydrological Processes, Vol. 30, No. 22, 2016, pp. 3991-4003. doi:10.1002/hyp. 10968.

[54] Saltelli, A., "Making best use of model evaluations to compute sensitivity indices," Computer Physics Communications, Vol. 145, 2002, pp. 280-297.

[55] Gilquin, L., Arnaud, E., Prieur, C., and Janon, A., "Making best use of permutations to compute sensitivity indices with replicated orthogonal arrays," Reliability Engineering and System Safety, Vol. 187, No. C, 2019, pp. 28-39. doi:10.1016/j.ress.2018.09.010. 